\title{
Burkholderia cepacia - outbreak in obstetric patients due to intrinsic contamination of non- sterile ultrasound gel
}

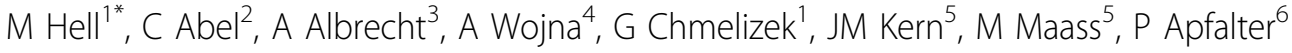 \\ From International Conference on Prevention \& Infection Control (ICPIC 2011) \\ Geneva, Switzerland. 29 June - 2 July 2011
}

\section{Introduction / objectives}

Ultrasound gel is a potential source of infection. Nonsterile ultra-sound gels can be contaminated due to manufacturing procedures and also during usage of opened bottles. We report a cluster of eight clinical cases of vaginal colonization (one clinically proven colpitis) with Burkholderia cepacia (B. cepacia) due to intrinsically contaminated ultrasound gel in obstetric patients in an Austrian hospital.

\section{Methods}

When the cluster was realised a microbiological investigation of the environment was initiated (e.g. surfaces, equipment, ultrasound gels of different manufacturers). Isolates of three different patients and four isolates of ultrasound gel bottles (two opened and two sealed) were investigated by Puls-Field-Gel-Electrophoresis (PFGE) to clarify clonality and source.

\section{Results}

Environmental specimens revealed no growth of B. cepacia. The four bottles from the incriminated manufacturer (two opened, two sealed bottles, all belonging to the same batch) were highly contaminated with B. cepacia (up to $40.000 \mathrm{CFU} / \mathrm{ml}$ ). These isolates and the three patients' isolates showed the same genotype pattern by PFGE.

\section{Conclusion}

We, therefore, concluded that the whole batch of these non-sterile gels was affected by monoclonal intrinsic contamination with B. cepacia due to insufficiently

'Dep.of Hospital Epidemiology and Infection Control, University Hospital

Salzburg, Salzburg, Austria

Full list of author information is available at the end of the article controlled manufacturing procedures. There is a need for discussion about microbiological contamination levels tolerated in such non-sterile ultrasound gels, especially when used on susceptible sites such as mucous membranes.

\section{Disclosure of interest}

None declared.

\section{Author details}

'Dep.of Hospital Epidemiology and Infection Control, University Hospital Salzburg, Salzburg, Austria. ${ }^{2}$ Infection control team, Hallein, Austria. ${ }^{3}$ Dep.of Gynecology, Teaching Hospital Hallein, Hallein, Austria. ${ }^{4}$ Private Laboratories Dr.Mustafa/Dr.Richter, Dep.of clinical microbiology, University Hospital Salzburg, Salzburg, Austria. ${ }^{5}$ Institute of medical microbiology, hygiene and infectious diseases, University Hospital Salzburg, Salzburg, Austria. ${ }^{6}$ Institute of Hygiene, Medical Microbiology and Tropical Medicine, Teaching Hospital Elisabethinen, Linz, Austria.

Published: 29 June 2011

\section{doi:10.1186/1753-6561-5-S6-075}

Cite this article as: Hell et al:: Burkholderia cepacia - outbreak in obstetric patients due to intrinsic contamination of non-sterile ultrasound gel. BMC Proceedings 2011 5(Suppl 6):075. 